\title{
No Laryngeal Vestibular Closure
}

National Cancer Institute

\section{Source}

National Cancer Institute. No Laryngeal Vestibular Closure. NCI Thesaurus. Code C127256.

A finding of no laryngeal vestibular closure; wide column air/contrast in laryngeal vestibule. 